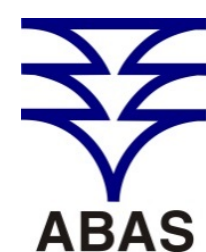

ASSOCIACÁOB BRASILERADE www.abas.org

\section{BALANÇO HÍDRICO E MODELAGEM COMPUTACIONAL VISANDO ESTIMAR RECARGA SUBTERRÂNEA}

\section{USING WATER BUDGET AND COMPUTATIONAL MODELING TO ESTIMATE GROUNDWATER RECHARGE}

\author{
Davi de Carvalho Diniz Melo ${ }^{1}$; Manoel Camilo Moleiro Cabrera²; \\ Edson Wendland ${ }^{3}$
}

Artigo recebido em: 27/07/2016 e aceito para publicação em: 05/12/2016.

DOI: http://dx.doi.org/10.14295/ras.v31i1.28656

\begin{abstract}
Resumo: As águas subterrâneas são a principal fonte hídrica em diversos países e regiões na América do Sul e Brasil. Entretanto, os volumes extraídos desses mananciais já ultrapassam a capacidade de reposição, resultando na diminuição dos níveis dos aquíferos. Assim, a garantia de disponibilidade futura depende do correto gerenciamento desses recursos hídricos. Para isso, é necessário conhecer as taxas de recarga e o fluxo subterrâneo que ocorrem nos aquíferos. Neste trabalho, avalia-se a recarga subterrânea para diferentes usos agrícolas com base no balanço hídrico da zona não saturada do solo. A avaliação foi feita, primeiramente, por meio da comparação com estimativas obtidas pelo método da Flutuação da Superfície Livre (WTF - Water Table Fluctuation). Em seguida, as taxas computadas foram usadas como entradas num modelo transiente de fluxo com intuito de verificar sua consistência por meio da comparação com dados de níveis medidos em campo. Os resultados indicam que o método aplicado apresentou desempenho satisfatório e, apesar de limitações inerentes, evidenciam a vantagem de sua utilização em relação a outras técnicas por requerer dados de maior facilidade de obtenção.
\end{abstract}

Palavras-Chave: Modelo SPA. Calibração. Fluxo transiente.

Abstract: Groundwater is the main water source in several countries and regions in South America and Brazil. However, the overexploitation in some regions exceeds the aquifer's replenishment capacity, resulting in the lowering of the water table. Thus, it is paramount that the use of these water resources are manage properly, assuring future availability. To that end, the groundwater flow and recharge rates need to be assessed. In this study, groundwater recharge estimated based on the water balance in the unsaturated zone is assessed. Firstly, this approach is evaluated by comparing its results with the Water Table Fluctuation method. Then, the recharge estimates are used as inputs in a transient groundwater flow model and resulting simulated water levels are compared to monitoring. The results suggest that the adopted approach leads to satisfactory results and, despite some inheriting limitations, it has the advantage of being less data demanding than other techniques.

Keywords: SPA model. Calibration. Transient flow.

\section{INTRODUÇÃO}

O Sistema Aquífero Guarani (SAG) é um dos mais importantes mananciais de água subterrânea da América do Sul, com volume estimado entre 25.000 e 37.000 $\mathrm{km}^{3}$ (OEA, 2009). Sua área distribui-se por territórios brasileiros $\left(736.000 \mathrm{~km}^{2}\right)$, argentinos (228.200 $\left.\mathrm{km}^{2}\right)$, paraguaios $\left(87.500 \mathrm{~km}^{2}\right)$ e uruguaios $\left(36.200 \mathrm{~km}^{2}\right)$ (GASTMANS et al., 2010). No Brasil, o SAG ocorre nos estados de Goiás, Mato Grosso, Mato Grosso do Sul, Minas Gerais, São Paulo, Paraná, Santa Catarina e Rio Grande do Sul. De acordo com Wendland \& Rabelo (2010), o SAG é essencialmente confinado ou semi-confinado, com áreas de afloramento, onde ocorre a recarga,

\footnotetext{
1,3 Universidade de São Paulo (USP), São Paulo, SP - E-mails: (melo.dcd@gmail.com, ew@sc.usp.br)

2 Universidade Estadual de Santa Cruz (UESC), Ilhéus, BA - E-mail: cabrera.uesc@gmail.com)
} 
correspondendo a aproximadamente 10\% da área total. No estado de São Paulo, aproximadamente $15 \%$ dos $155.800 \mathrm{~km}^{2}$ correspondem à zona de afloramento do SAG. É também nesse estado onde se concentra a maior quantidade de poços, representando 90\% da extração de água em território brasileiro (OEA, 2009).

$\mathrm{O}$ entendimento dos mecanismos de recarga e do fluxo de água subterrânea nesse sistema é de fundamental importância para o gerenciamento sustentável de seus recursos hídricos. Entretanto, a recarga subterrânea é um dos componentes do ciclo hidrológico mais difícil de ser obtida, uma vez que não há métodos de medição direta da mesma. Diversas técnicas são descritas na literatura, como por exemplo, lisímetros, traçadores e balanço hídrico mas todas apresentam limitações e incertezas, tornando importante a comparação entre estimativas obtidas por diferentes métodos (SCANLON et al., 2006). Dentre estes, o balanço hídrico oferece a vantagem de sua aplicação não ser limitada a apenas uma das zonas do solo (Healy et al., 2007).

A modelagem computacional dos mecanismos de recarga e fluxo possibilita o entendimento dos processos e fornece informações que podem ser utilizadas para auxiliar no processo de tomada de decisão (Wendland \& Rabelo, 2010). No entanto, é necessário que as estimativas de recarga e simulação do fluxo sejam validadas para as condições que efetivamente ocorrem no Sistema Aquífero Guarani.

Para realizar essa validação, este trabalho utilizou dados medidos em campo em uma das áreas com características representativas da zona de recarga do SAG: a bacia do Ribeirão da Onça (BRO). Nessa bacia, o grupo de pesquisa do Laboratório de Hidráulica Computacional (LHC) realiza continuamente o monitoramento quinzenal do nível d’água subterrâneo (Lucas et al., 2012), disponibilizando os dados para a estimativa da recarga (http://www.lhc.shs.eesc.usp.br/dadoshidr ometeorologicos/). Tendo como objetivo principal a validação do método do balanço hídrico (MBH), as estimativas assim obtidas foram verificadas pela análise da resposta de um modelo computacional transiente de fluxo, construído para a área de estudo.

\section{MATERIAIS E MÉTODOS}

A bacia experimental do Ribeirão da Onça está localizada no município de Brotas - SP, entre os paralelos $22^{\circ} 10^{\prime}$ e $22^{\circ}$ $15^{\prime} \mathrm{S}$ e entre os meridianos $47^{\circ} 55^{\prime}$ e $48^{\prime}$ 00’ O (Figura 1). O Ribeirão da Onça é um dos contribuintes para a formação do rio Jacaré-Guaçú, afluente do rio Tietê. Uma de suas características que gera maior interesse é sua localização em área de afloramento do SAG.

Próximo do centro da BRO ( 7 km) encontra-se o Centro de Recursos Hídricos e Ecologia Aplicada (CRHEA/USP), onde está instalada uma estação climatológica (EC) convencional. Nesta estação foram medidas as variáveis meteorológicas, verificando que a região apresenta temperatura e umidade relativa média (1974-2014) de $21,6{ }^{\circ} \mathrm{C}$ e $71 \%$, respectivamente. $\mathrm{O}$ mês de fevereiro apresenta a maior temperatura média 24,3 ${ }^{\circ} \mathrm{C}$, junho e julho são os meses com as menores temperaturas médias $\left(17,7^{\circ} \mathrm{C}\right)$. $\mathrm{O}$ acumulado médio mensal de chuva em julho e agosto é o menor, sendo apenas 30 $\mathrm{mm}$. Agosto é o mês com a menor umidade relativa média, com $64 \%$, e a maior taxa de insolação com 263 horas/mês. O mês mais nublado (167 horas de insolação por mês), úmido (UR $\left.\mathrm{Umd}_{\mathrm{med}} 77 \%\right)$ e chuvoso $(271 \mathrm{~mm})$ é janeiro. A precipitação média anual, entre 1938 e 2015, foi de $1470 \mathrm{~mm}$. A chuva acumulada média mensal medida é mostrada na Figura 2. 


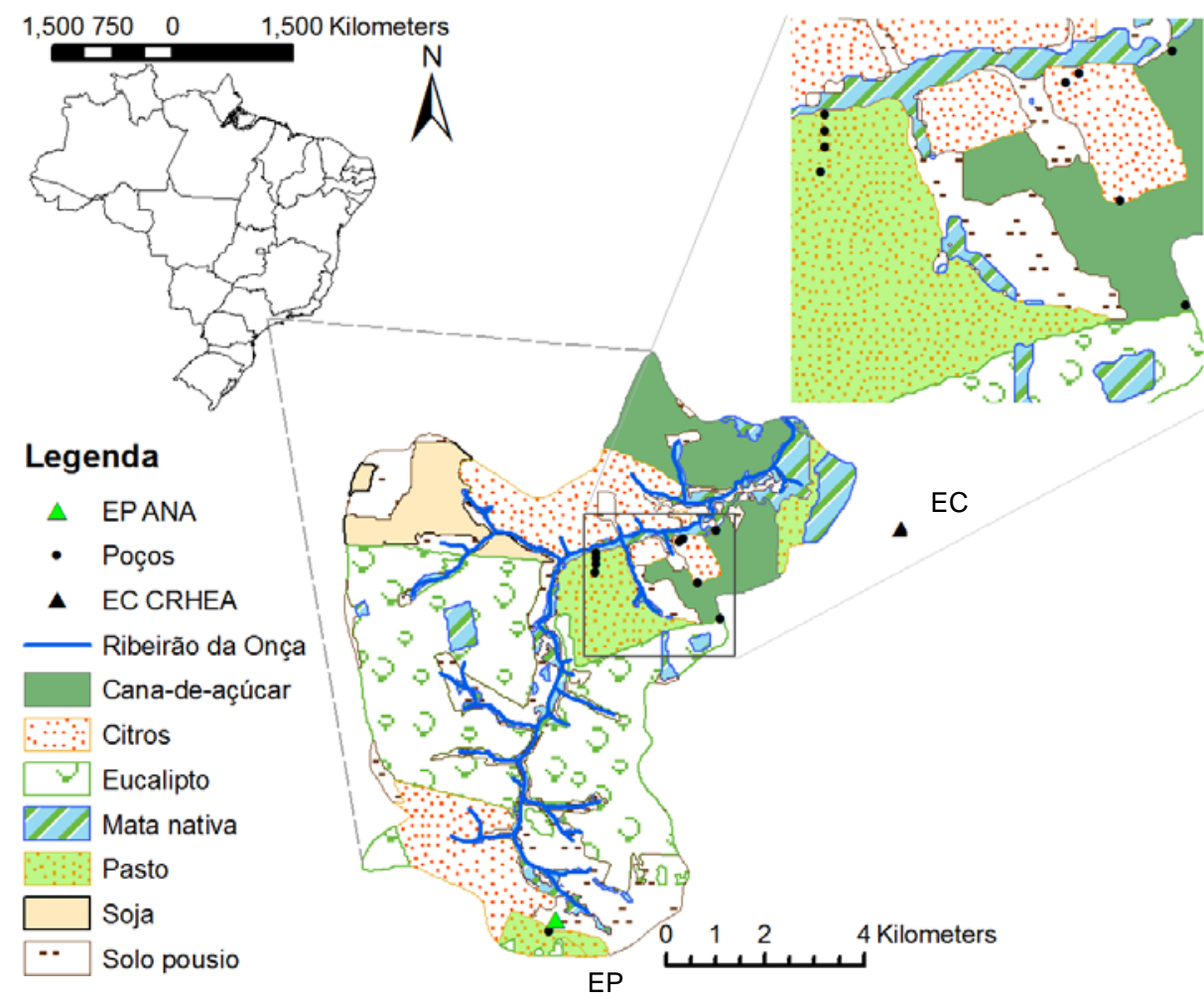

Figura 1 - Área de estudo: bacia hidrográfica do Ribeirão da Onça e mapa de uso do solo referente ao período analisado (2004-2014). EP = Estação pluviométrica; EC = Estação climatológica

Figure 1 - Study area: Onça Creek basin and land use map for the analyzed period (2004-2014). EP $=$ Rain gauge $\mathrm{EC}=$ climatological gauge

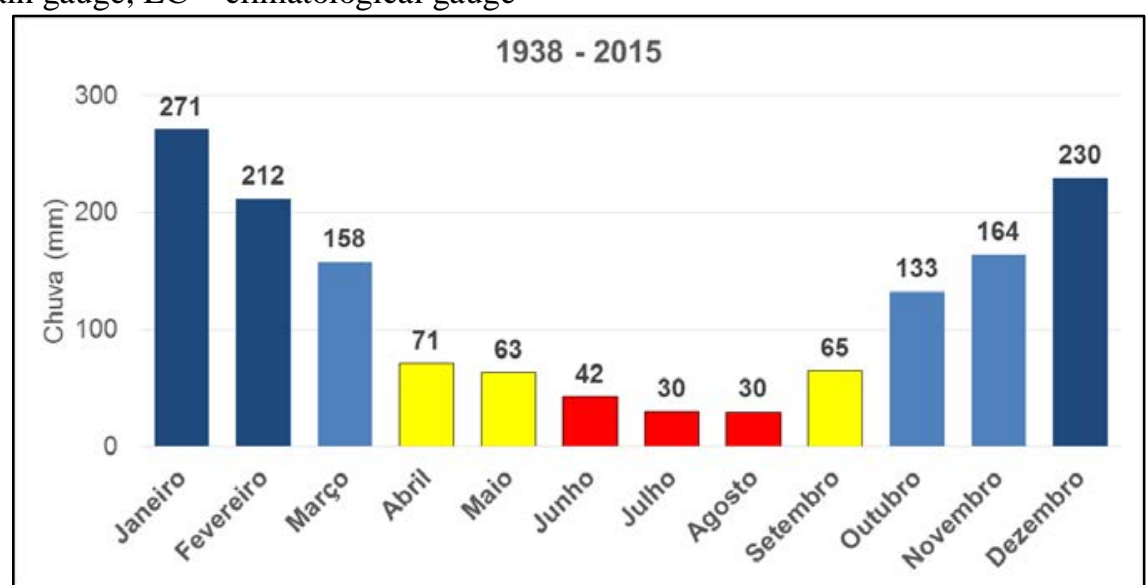

Figura 2 - Chuva acumulada média mensal entre 1938 e 2015. Os dados foram coletados próximo ao exutório da bacia nos postos pluviométricos da Agência Nacional de Águas ANA (2247196 CRHEA-BROA) e Departamento de Águas e Energia Elétrica (D4-033 Usina do Lobo)

Figure 2 - Mean monthly rainfall between 1938 and 2015. The data were colleted near the basin outlet from the rain gauges belonging to National Water Agency - ANA (2247196 CRHEA-BROA), and Water and Electric Energy Department of São Paulo state (D4-033 Usina do Lobo)

O clima da região, de acordo com a classificação de Wilhem Köppen, é Cwa (clima temperado) devido à altitude, com inverno seco, apresentando pouca deficiência de água (MACHADO e MATTOS, 2001). Os principais usos do solo identificados são: pastagem, cana-deaçúcar, eucalipto, citros (laranja ou limão), soja e mata nativa de Cerrado. O balanço hídrico climatológico normal indica que o maior saldo positivo ocorre em janeiro (171 mm), enquanto que em agosto o déficit é de $5 \mathrm{~mm}$ (Figura 3). A evapotranspiração potencial mensal para o balanço hídrico normal foi estimada com a equação de Thornthwaite. 


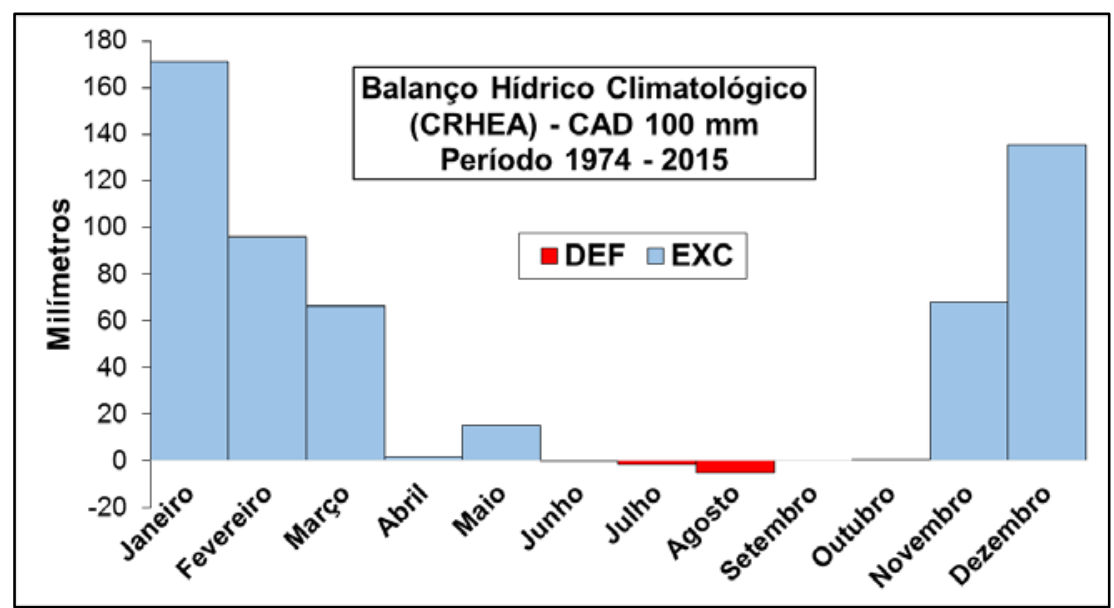

Figura 3 - Balanço hídrico climatológico (1974-2015) para a região

Figure 3 - Climatological water budget (1947-2015) for the study area

Os dados de precipitação e temperatura (2004-2014) foram obtidos na estação climatológica do CRHEA. Também foram utilizados os dados de precipitação da estação da ANA (2247197 Campo Alegre), localizada no sul da BRO (Figura 1). Os dados de nível freático do aquífero livre (NE) foram medidos em poços de monitoramento instalados em parte da bacia (Figura 1). Outros dados de caráter hidrogeológico, necessários à construção do modelo, são: condutividade hidráulica, porosidade e espessura do aquífero. A determinação da porosidade do aquífero na BRO foi determinada por Gomes (2008) a partir de análises de amostras indeformadas, coletadas em cinco pontos dentro da BRO e em três profundidades que variaram de 3 a $19 \mathrm{~m}$. Para realizar essa análise, Gomes (2008) projetou um amostrador e acoplou junto a um equipamento de perfuração. Em seguida, ele utilizou o método do funil de Haines e da saturação com drenagem para analisar a porosidade. Em cada ponto, as profundidades correspondem à respectiva faixa de variação do nível freático.

Dados planialtimétricos e de drenagem foram obtidos a partir de mapas de drenagem fluvial com as respectivas curvas de nível e de imagens da Shuttle Radar Topographic Mission (SRTM), com resolução de $30 \mathrm{~m}$. Visitas a campo foram feitas para obtenção de cotas altimétricas de algumas nascentes dos afluentes do Ribeirão da Onça. A imagem da SRTM foi processada em Sistema de Informação Geográfica (SIG) para produzir o modelo digital de elevação (MDE) da área de estudo.

A área de estudo possui unidade hidroestratigráfica complexa tornando a representação numérica da região não confinada do aquífero uma ferramenta essencial na modelagem e simulação do fluxo de água subterrânea. O modelo conceitual adotado admite que não ocorram regiões de confinamento na BRO. As fronteiras do modelo subterrâneo correspondem aos divisores topográficos da bacia e nelas aplicaram-se condições do $2^{\circ}$ Tipo (Neumann). Este tipo de condição possibilita a especificação do fluxo por determinada área, sendo que nos divisores de água o fluxo é considerado nulo. À rede de drenagem foi aplicada a condição de $3^{\circ}$ Tipo (Robin).

O modelo numérico foi construído usando o pacote computacional SPA Simulador de Processos em Aquíferos (Wendland, 2003). Esse software possibilita a simulação em regime permanente e transiente de problemas de fluxo de água subterrânea e de transporte de solutos em domínios tridimensionais em meios porosos, saturados e não-saturados. O modelo usa o método dos elementos finitos (MEF) para discretizar o domínio e 
solucionar o conjunto de equações de fluxo.

Um parâmetro indicador da qualidade da modelagem corresponde ao cálculo do erro quadrático médio normalizado entre as cargas simuladas e

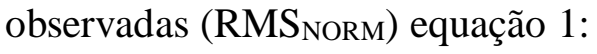

$$
\begin{aligned}
& R M S_{N O R M} \\
& =\frac{\frac{\sqrt{\sum(O-S)^{2}}}{n}}{\bar{O}}
\end{aligned}
$$

(O) valor observado; (S) valor simulado; (n) número de observações e $(\bar{O})$ média aritmética das observações.

A recarga subterrânea foi estimada pelo balanço hídrico e comparada com os resultados apresentados por Lucas et al. (2012), que utilizaram o método da variação da superfície livre (Water Table Fluctuation - WTF) (Healy \& Cook, 2002). A recarga foi estimada considerando os diferentes usos do solo apresentados na Figura 1. A recarga mensal (R) foi calculada como uma parcela da recarga potencial mensal $\left(\mathrm{R}_{\mathrm{p}}\right)$ (Melo, 2013), dada pela parte residual do balanço na camada superficial, correspondente à zona de raízes do solo (Equação 2):

$$
R_{P}=P-E T_{r}-E s-\Delta S_{z n s}
$$

Em que $\mathrm{P}$ é a precipitação mensal, $E T_{r}$ é a evapotranspiração real, $E s$ é o escoamento superficial e de base, $\Delta S_{z n s}$ é a variação do armazenamento na zona nãosaturada. A precipitação usada no balanço é dada pela média ponderada entre as medições das estações da Agencia Nacional de Águas CRHEA-BROA 2247196 e Campo Alegre 2247197, tendo como pesos as distâncias entre a estação e a área da cobertura vegetal em questão.

A estimativa de ETr, descrita detalhadamente por Melo (2013), foi obtida a partir da evapotranspiração potencial corrigida $\left(\mathrm{ETP}_{\mathrm{cor}}\right)$ e de valores de $\mathrm{K}_{\mathrm{c}}$ médio por cultura (Allen et al, 1998). ETP $_{\text {cor }}$ foi obtida pela correção, proposta por Medeiros et al. (2012), da evapotranspiração potencial calculada pela equação de Thornthwaite.

$\mathrm{Na}$ área de estudo, dados de escoamento superficial, medidos experimentalmente, inexistem para o período analisado. Os dados de Es usados neste trabalho são oriundos do modelo hidrológico da BRO calibrado por Machado et al., (2016) utilizando o sistema de modelagem JAMS.

A variação do armazenamento na zona não-saturada foi computada pela diferença entre o conteúdo de água no solo do mês avaliado e do mês anterior. $\mathrm{O}$ modelo do balanço hídrico implementado por Melo (2013) considera que os diferentes usos do solo dispõem de uma capacidade máxima de retenção de água que depende do alcance das raízes dentro da zona não-saturada $\left(S_{\max }\right)$. A precipitação (P) consiste na única entrada de água do balanço hídrico, o saldo de água que entrará no reservatório causará o aumento ou diminuição do nível de água no solo.

\section{RESULTADOS}

\subsection{Modelo computacional}

A malha de EF foi gerada com espaçamento mínimo de 200 metros e refinamento na proximidade na proximidade de rios e poços. A espessura da camada saturada modelada é variável, uma vez que a cota da base do modelo oscila de $\sim 630$ a $670 \mathrm{~m}$ (Guanabara 2011). A espessura média dessa camada é de 50 m. Para iniciar a simulação transiente, o modelo foi alimentado com a superfície potenciométrica criada a partir da interpolação dos níveis freáticos medidos. Após a simulação transiente, os resultados apresentados na Figura 4 foram analisados. A Figura 4 (a) representa o mapa de fluxo na BRO, tendo as cargas hidráulicas simuladas em cada elemento representadas por vetores. A distribuição espacial de velocidade confirma a consistência do modelo, uma vez que não foram 
identificadas regiões com valores irreais de escoamento nem com mudanças bruscas de direção.

Os parâmetros de condutividade hidráulica e porosidade foram ajustados manualmente com base na comparação entre os valores de carga hidráulica e vazão de base simulados e medidos durante o período compreendido entre 2004-2011 (calibração) e 2011-2014 (validação). Os valores iniciais desses parâmetros foram baseados em estimativas apresentadas por Sracek \& Hirata (2002) e Gastmans \& Kiang (2005).

a)

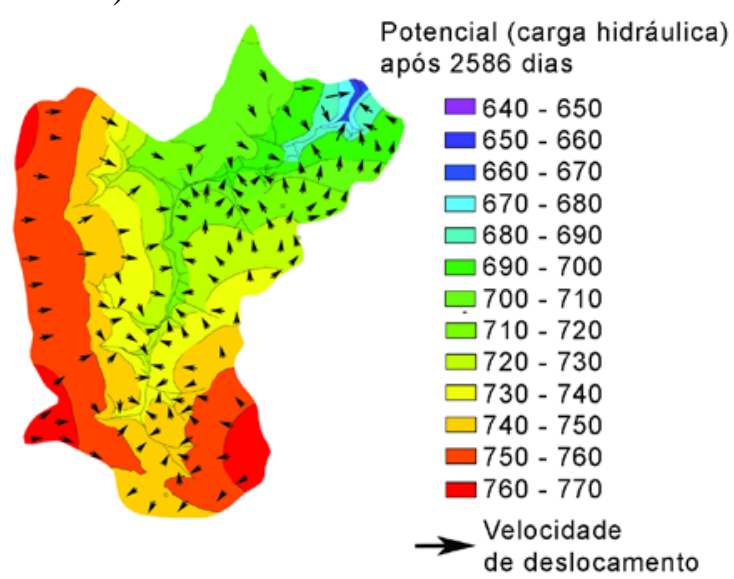

c)

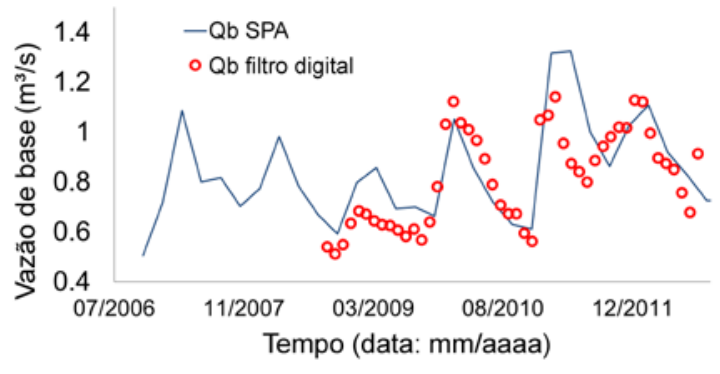

Baseando-se em dados de solo, geologia, hidrogeologia e estimativas apresentadas para outras áreas do SAG por Sracek \& Hirata (2002) e Gastmans \& Kiang (2005), adotou-se o valor inicial de $\mathrm{K}=4,32 \mathrm{~m} \mathrm{dia}^{-1}\left(5 \times 10^{-5} \mathrm{~m} \mathrm{~s}^{-1}\right)$. Essa estimativa inicial foi modificada ao longo do processo de calibração e espacializada com base no mapa pedológico da área. Os valores finais de $\mathrm{K}\left(1,3 \times 10^{-6}\right.$ a $2,6 \times 10^{-5} \mathrm{~m}$ $\left.\mathrm{s}^{-1}\right)$ possuem ordem de grandeza dentro do intervalo daqueles encontrados por Sracek \& Hirata (2002) $\left(2 \times 10^{-6} \mathrm{~m} \mathrm{~s}^{-1}\right.$ a $1,9 \times 10^{-3} \mathrm{~m}$ $\left.\mathrm{s}^{-1}\right)$ no estado de São Paulo.

\section{b)}

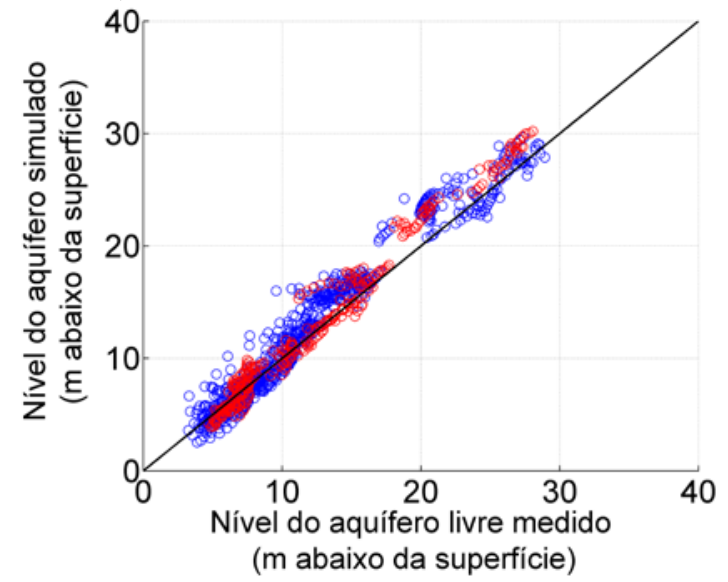

d)

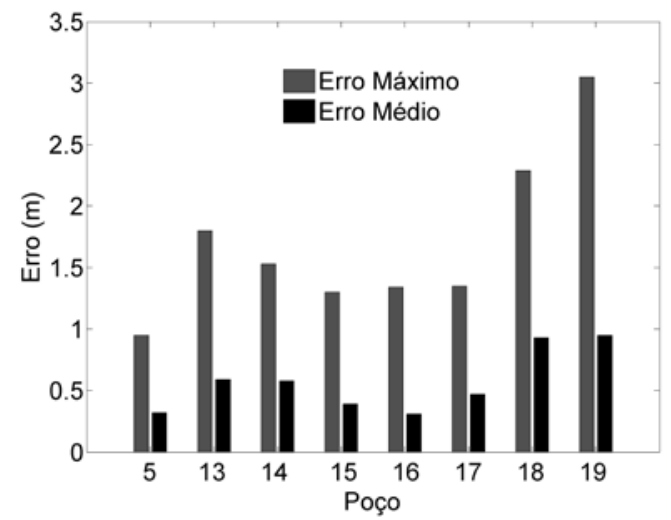

Figura 4 - Resultados da simulação: mapa de fluxo (a), níveis medidos e simulados na calibração (em azul) e validação (em vermelho) do modelo, (c) vazão de base simulada pelo modelo e calculada pelo filtro digital, (d) erros máximos e médios nos nós referentes aos poços de monitoramento

Figure 4 - Simulation results: flux map (a), simulated and observed groundwater levels from model calibration (blue) and validation (red), (c) baseflow simulated by the model and derived from the digital filter, (d) maximum and mean errors in the nodes relative to the monitoring wells

Uma estimativa dos valores de rendimento específico envolvidos na simulação é dada por Gomes (2008) para a área de estuodo variam entre 8,5 e 15,9 \%.
Esses resultados se assemelham aos encontrados por Sracek \& Hirata (2002) para regiões do SAG (7 a 15\%). Para espacializar a porosidade considerou-se as 
áreas de influência definidas por Gomes (2008), obtidas em função da espessura da camada não-saturada média e da distância dos pontos de coleta ao curso d'água.

Os resultados da calibração (azul) e validação (vermelho) do modelo mostram boa concordância entre os níveis simulados e observados nos poços de monitoramento (Figura 4b). Os poços 13, 16, 17 e 19

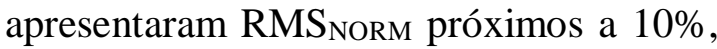
sendo que os poços 13, 17 e 19 este parâmetro foi de 9 , 10 e $11 \%$. O poço 16 apresentou $\mathrm{RMS}_{\text {NORM }}$ igual a $13 \%$, apesar de ser o maior valor entre os quatro poços, é um resultado bom face a dificuldade de modelagem do nível em zonas riparias e de transição de vegetação. Na composição do RMS $_{\text {NORM }}$ foi utilizado 114 pares mensais de cargas simulados e observados.

A comparação entre a vazão de base fornecida pelo SPA e aquela obtida por meio da aplicação do filtro digital recursivo proposto por Nathan \& MacMahon (1990), indica que o balanço de massa do modelo computacional é consistente com as condições observadas em campo (Figura 4c). Esta comparação permitiu testar a boa qualidade da vazão de base modelada frente à um método consagrado na literatura, fazendo com que o erro médio fosse igual ou abaixo de 1,0 metro (Figura 4c) A Para cada nó correspondente aos poços de monitoramento, foram gerados relatórios de desvio pelo software (Figura 4d), sendo indicados os desvios médios e máximos referentes a todo período de simulação.

$\mathrm{O}$ segundo ano da simulação apresentou maior dificuldade para calibração, pois os níveis observados quase não respondem aos eventos de precipitação, atingindo uma diferença máxima de 1,80 m em relação ao simulado no P13. Para esse nó, o erro médio (0,59 m) foi ligeiramente superior ao encontrado para o P14 (0,58 m) (Figura 4d). Essas diferenças podem indicar que as taxas de recarga calculadas para os respectivos períodos estão sub ou superestimadas pelo método adotado.

Apesar dos ajustes feitos, o modelo continua sendo uma simplificação dos processos reais e, portanto, contém limitações que, neste estudo, puderam ser identificadas próximas às condições de contorno. Um exemplo é o nó referente ao poço 16 (P16), que se encontra na fronteira entre elementos da malha referentes à mata nativa (Mata) e ao Pasto. Esta posição pode gerar uma incerteza sobre a influência de cada vegetação no nível de água.

No período chuvoso, o nível do aquífero eleva-se até próximo da superfície ou aflora nas áreas marginais do rio. Com isto, as perdas por evaporação direta elevam-se (verão) e diante da complexidade em serem incorporadas no balanço hídrico para estimativa da recarga, o modelo responde por meio de um efeito de represamento, expresso na forma de picos acima do observado. Ainda assim, para o restante do período, os níveis simulados se aproximam satisfatoriamente dos medidos, com erro médio inferior a 0,5 $\mathrm{m}$ nos poços 16 e 17 .

\subsection{Níveis de água subterrânea e chuva}

Selecionaram-se quatro poços para visualização do comportamento temporal dos níveis de água subterrânea medidos e simulados. A chuva acumulada mensal no período de análise também é mostrada em conjunto com a variação dos níveis de água (Figuras 5 a 8). 


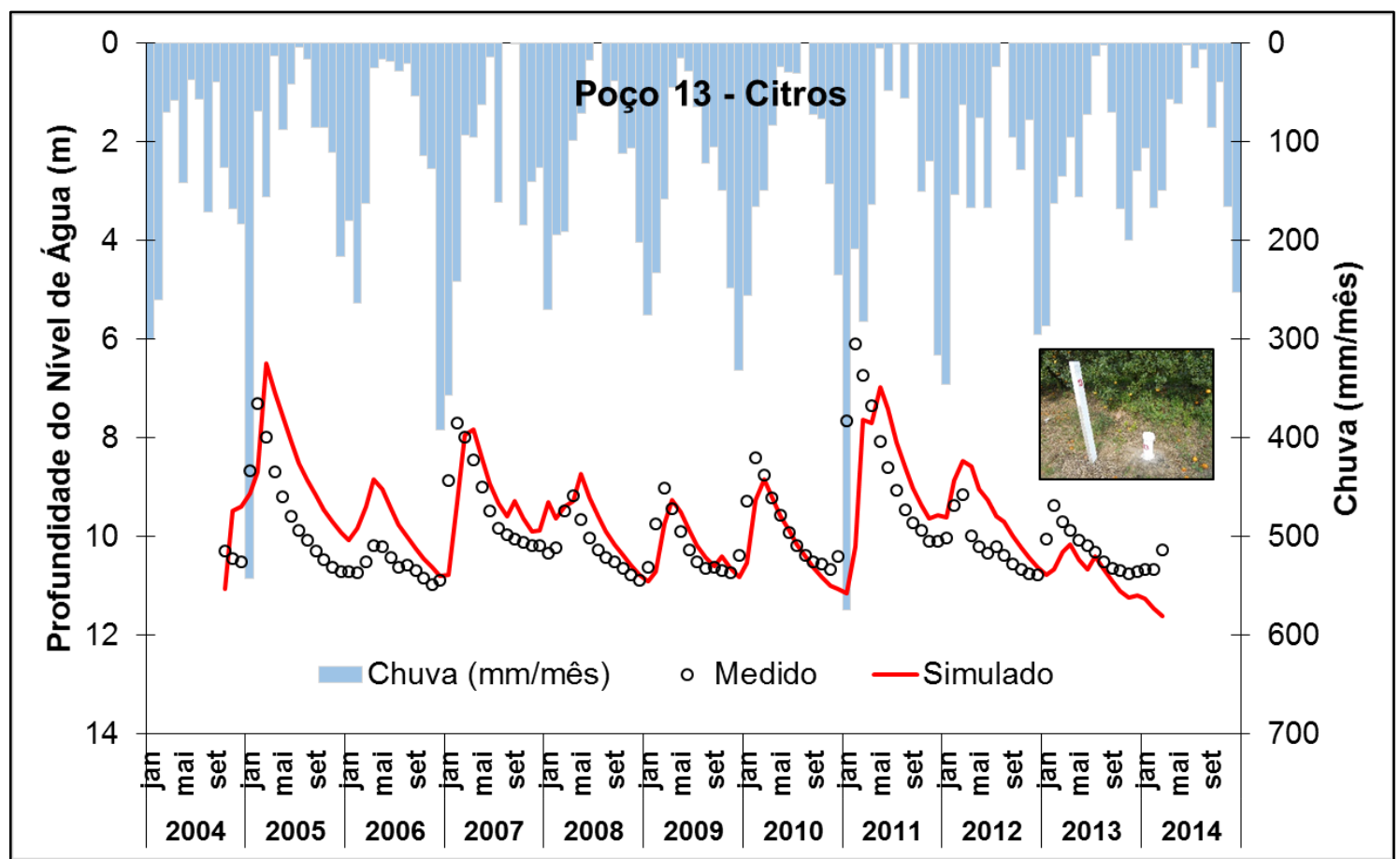

Figura 5 - Nível de água subterrânea medido (círculos) e simulado (linha vermelha) no poço 13 durante final de 2004 e início de 2014. Neste período o solo foi coberto por pomar de citros. O acumulado de chuva mensal (CRHEA-BROA 2247196) também é indicado por barras verticais

Figure 5 - Simulated (red lines) and observed (black circles) groundwater levels for well 13 between 2004 and 2014, and monthly rainfall (vertical bars). During this period, the land use around the well consisted of citrus plantings

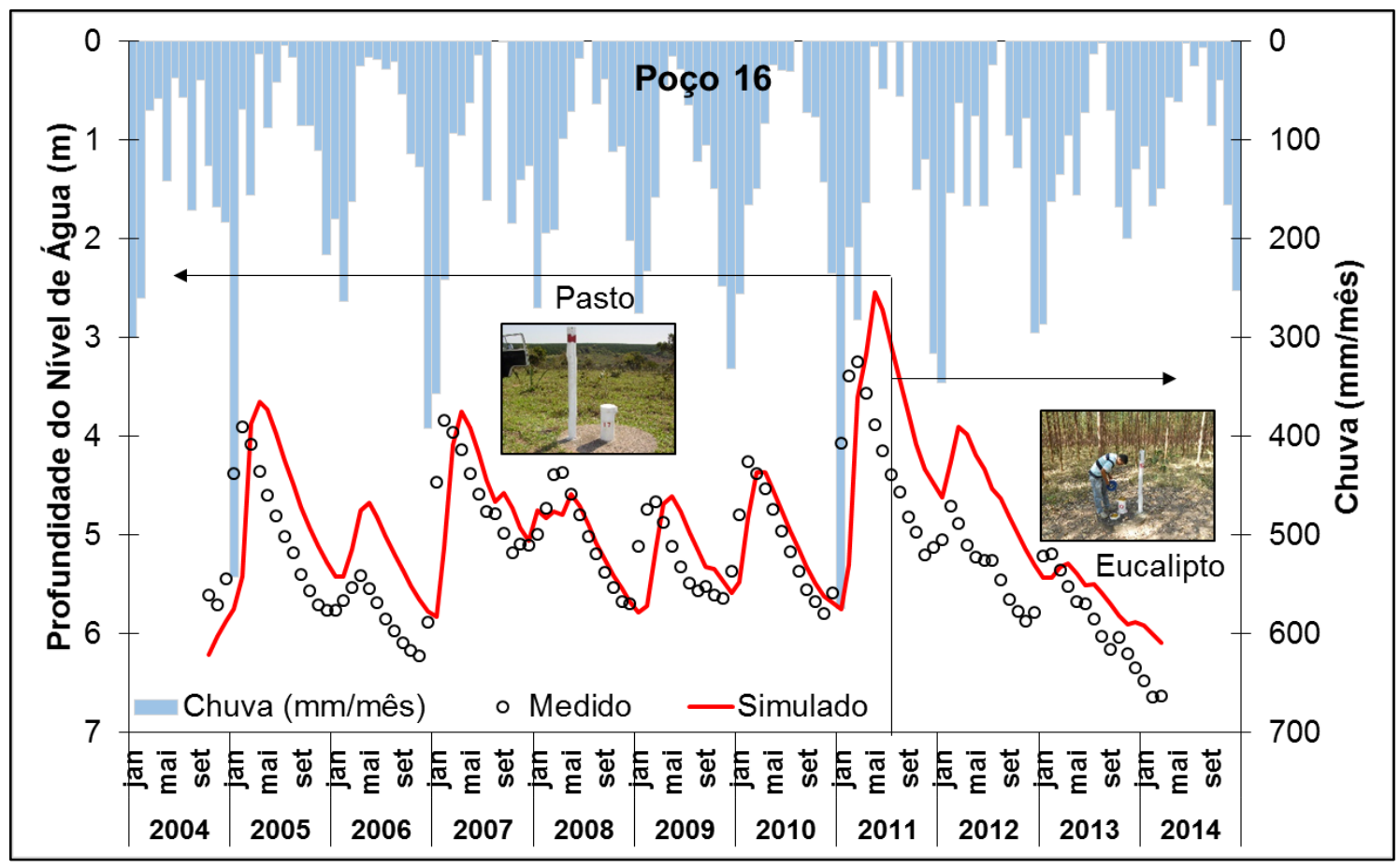

Figura 6 - Nível de água subterrânea medido (círculos) e simulado (linha vermelha) no poço 16 durante final de 2004 e início de 2014. Até 2011 o solo foi coberto por pastagem, após este período a cobertura foi de eucalipto. O acumulado de chuva mensal (CRHEA-BROA 2247196) também é indicado por barras verticais

Figure 6 - Simulated (red lines) and observed (black circles) groundwater levels for well 16 between 2004 and 2014, and monthly rainfall (vertical bars). From 2004 to 2011, land use around the well consisted of pasture, which was replaced by eucalyptus after 2011 


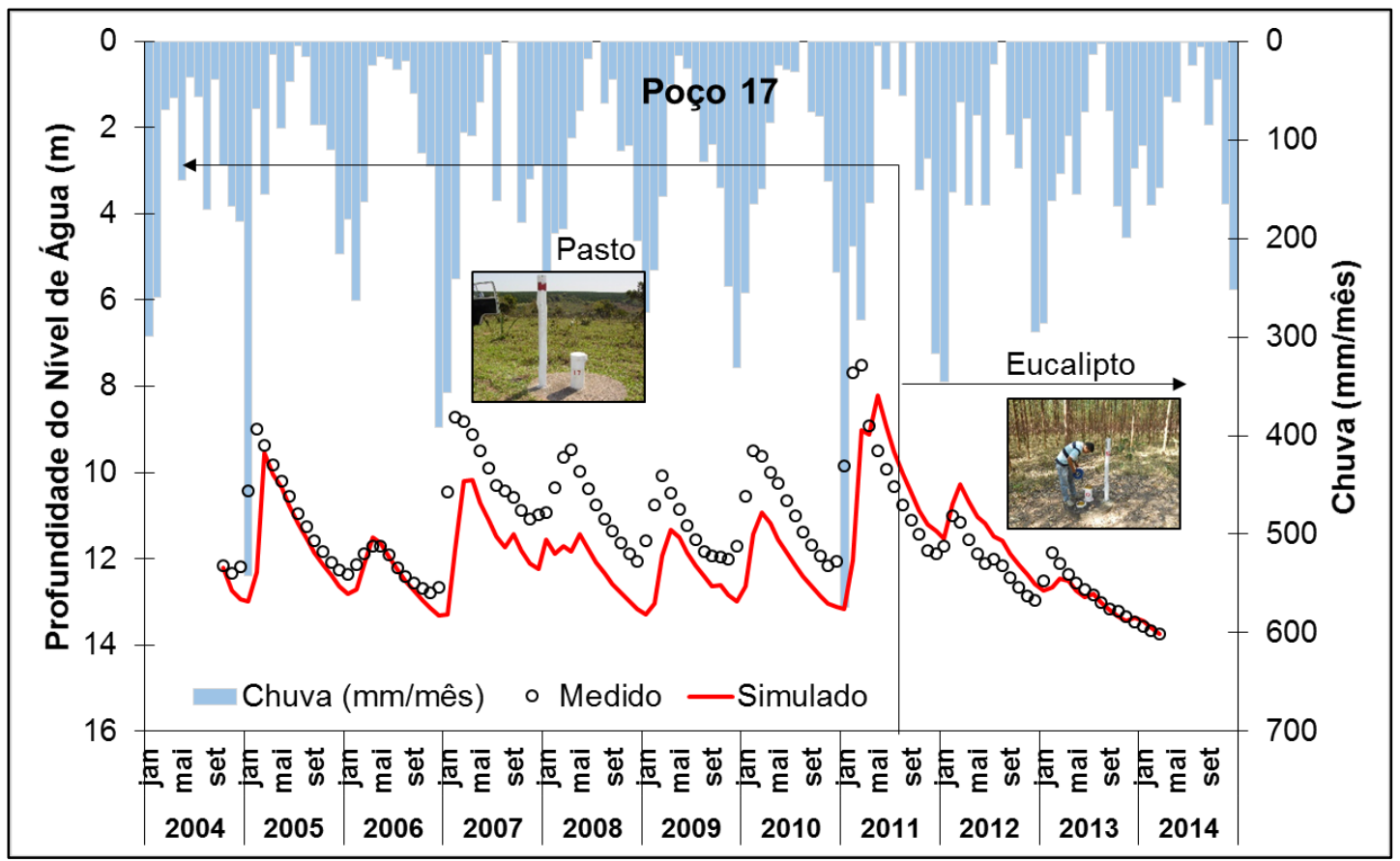

Figura 7 - Nível de água subterrânea medido (círculos) e simulado (linha vermelha) no poço 17 durante final de 2004 e início de 2014. Até 2011 o solo foi coberto por pastagem, após este período a cobertura foi de eucalipto. O acumulado de chuva mensal (CRHEA-BROA 2247196) também é indicado por barras verticais

Figure 7 - Simulated (red lines) and observed (black circles) groundwater levels for well 17 between 2004 and 2014, and monthly rainfall (vertical bars). From 2004 to 2011, land use around the well consisted of pasture, which was replaced by eucalyptus after 2011

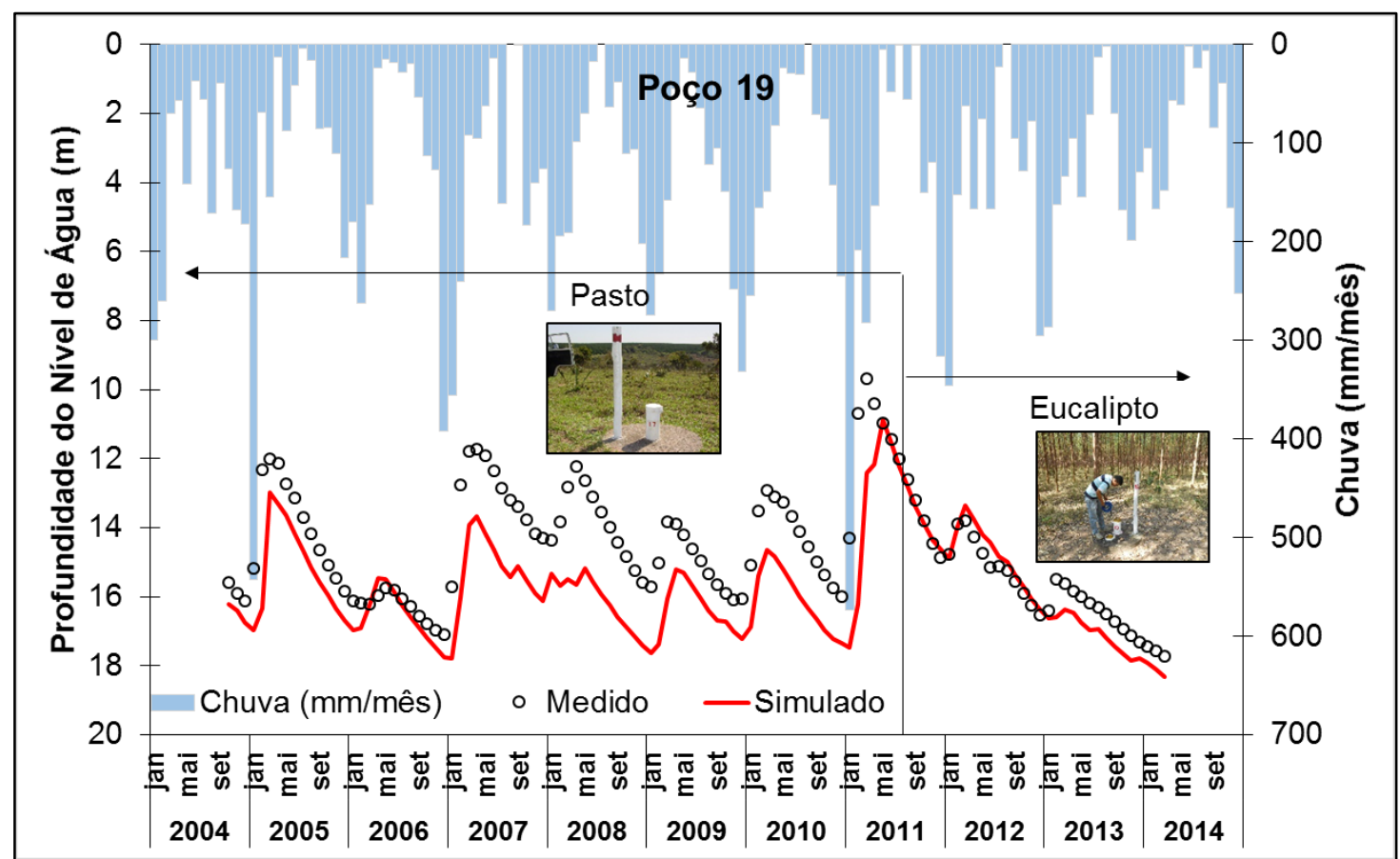

Figura 8 - Nível de água subterrânea medido (círculos) e simulado (linha vermelha) no poço 19 durante final de 2004 e início de 2014. Até 2011 o solo foi coberto por pastagem, após este período a cobertura foi de eucalipto. O acumulado de chuva mensal (CRHEA-BROA 2247196) também é indicado por barras verticais

Figure 8 - Simulated (red lines) and observed (black circles) groundwater levels for well 19 between 2004 and 2014, and monthly rainfall (vertical bars). From 2004 to 2011, land use around the well consisted of pasture, which was replaced by eucalyptus after 2011 
A cobertura vegetal nas imediações dos poços 17 e 19 em 2011 passou de pastagem para eucalipto (Figura 7 e Figura 8). Ao termino do verão 2012/2013 houve uma pequena elevação do nível nos poços 17 e 19, apesar da chuva ocorrida considerada média. Para o verão 2005/2006 a elevação do nível de água foi próxima a de 2012/2013 porém um pouco menor, o que era esperado, pois choveu abaixo da média. Esta diferença de pluviometria pode indicar que o eucalipto nos primeiros anos diminui a recarga, possivelmente devido a maior evapotranspiração. Dentre os quatro poços avaliados, estes apresentaram os níveis mais profundos. Os poços 19, 17 e 16 encontram-se alinhados. $\mathrm{O}$ poço 16 devido à proximidade do Ribeirão da Onça apresenta o nível de água mais raso (Figura 6). Este poço situa-se em uma zona de transição entre o pasto/eucalipto e a zona ripária. Dentre os quatro poços apenas o poço 13 manteve a mesma cobertura vegetal (citros) durante o intervalo de análise (Figura 5).

Nestes poços os níveis de água subterrânea mais rasos aconteceram durante o ano de 2011 devido à pluviometria extrema deste ano. Em 2011 ocorreu o quarto maior acumulado anual de chuva (1924 mm) da série histórica de 78 anos (1938-2015). Em janeiro de 2011 foi registrado o segundo maior acumulado de chuva mensal desta série (574 mm).

Os níveis mais profundos aconteceram no início de 2014 devido à baixa precipitação registrada em dezembro/2013 e janeiro/2014. Estes são os meses que apresentam as maiores precipitações e juntos correspondem a 34\% do total médio anual. Dezembro/2013 choveu apenas $56 \%$ do esperado para o mês $(230 \mathrm{~mm})$ e Janeiro/2014 39\% da média mensal (271 mm).

No geral os níveis de água subterrânea ficam mais próximos da superfície entre fevereiro e maio, aproveitando os maiores excedentes hídricos dos meses de janeiro e dezembro. Os níveis mais afastados da superfície acontecem nos meses de novembro e dezembro. Indicando que a elevação do nível de água do aquífero apresenta um atraso em relação aos primeiros meses com excedente hídrico após o intervalo de déficit no balanço hídrico climatológico (Figura 3).

\subsection{Estimativa de recarga}

As taxas de recarga calculadas em escala mensal para os diferentes tipos de uso do solo foram acumuladas anualmente com intuito de comparar com as estimativas feitas pelo método do WTF por Lucas et al. (2012). A referida comparação é mostrada na Figura 9, onde as maiores diferenças são observadas entre as estimativas para as áreas de pastagem e plantio dos anos de 2009 ( 100mm) e 2010 ( 120mm), respectivamente. Uma possível explicação para essas discrepâncias é a que a recarga depende da conformação topográfica e proximidade das zonas de recarga, estas podem resultar em variações significativas da subida do nível de água e por consequência a recarga estimada pelo WTF.

As diferenças entre os valores de recarga estimados pelo método do WTF e do BH podem ser explicadas pela incerteza relacionada a variáveis de entrada dos métodos. No caso do WTF, um parâmetro essencial na equação é o rendimento específico $\left(\mathrm{S}_{\mathrm{y}}\right)$ relacionado à porosidade do aquífero. Esse parâmetro é de difícil determinação e, em geral, varia ao longo da profundidade, devido à heterogeneidade de solos naturais. No caso do $\mathrm{BH}$, a variável de maior incerteza é a evapotranspiração (ET), cuja determinação depende de variáveis climáticas e de coeficientes de cultura $\left(\mathrm{K}_{\mathrm{c}}\right)$, adotado a partir de valores citados na literatura. DINGMAN (2014) cita que entre as variáveis do balanço hídrico a evapotranspiração é a mais difícil de se medir. 


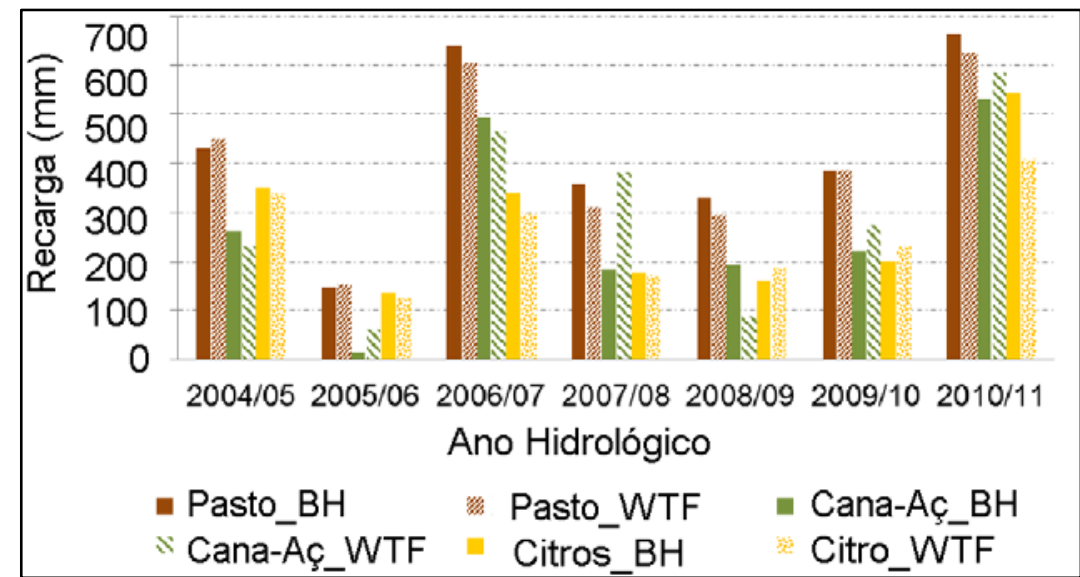

Figura 9 - Taxa de recarga estimada pelo método do balanço hídrico (MBH) e pelo método WTF Figure 9 - Recharge rates estimated by the water balance and WTF methods

\section{DISCUSSÕES}

Os resultados do modelo indicam boa relação entre os níveis simulados e observados nos poços de monitoramento. Apesar da heterogeinadade dos processos hidrológicos envolvidos, um exemplo disso são: a variação das taxas pluviométricas aliadas com mudança de cobertura do solo e fisiografia dos poços; o modelo computacional e suas simplificações apresenta resultados confiáveis. A vazão de base fornecida pelo SPA e aquela estimada com filtro digital recursivo proposto por Nathan \& MacMahon (1990), indica que o balanço hídrico feito com o modelo computacional é consistente com as condições de campo.

Em casos em que o sistema rioaquífero é do tipo hidraulicamente conectado (como é o caso da área de estudo), a vazão de base pode ser utilizada para estimar a recarga média na bacia. No entanto, essa abordagem fornece estimativas de recarga média na bacia, não sendo possível identificar como a recarga ocorre espacialmente. Por essa razão, tais estimativas não foram abordadas no presente trabalho.

A métrica estatística utilizada (RMS $_{\text {NORM }}$ ) permitiu visualizar e avaliar a qualidade da simulação em comparação com os valores medidos em campo. Constatou-se que o valor de RMS $_{\text {NORM }}$ próximo à 10\% comprova a boa estimativa do modelo em condições de mudança do uso do solo e situações extremas de pluviosidade. Mesmo em um poço localizado em área transicional de mata ciliar para eucalipto, sujeito a rápidas variações de nível devido a sua proximidade com o talvegue, o valor do

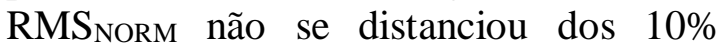
ficando apenas $3 \%$ acima deste.

\section{CONCLUSÕES}

A estimativa de recarga por meio do balanço hídrico apresentou resultados semelhantes àqueles obtidos utilizando 0 método do WTF. As diferenças entre os métodos estão possivelmente relacionadas às limitações intrínsecas dos métodos, variabilidade espacial da precipitação e incertezas nos dados de entrada. Embora a recarga subterrânea não possa ser diretamente medida para validar as estimativas obtidas indiretamente, o uso de um modelo computacional possibilita realizar essa verificação ao comparar níveis simulados com medições em campo. O modelo computacional também fornece informações que possibilitam melhor entendimento dos processos que ocorrem na área de estudo, ou ainda avaliar impactos de cenários de mudanças climáticas.

A abordagem proposta se destaca diante de outros métodos pelo fato de demandar dados de entrada mais facilmente obtidos. Por exemplo, método do WTF requer dados de rendimento 
específico da camada saturada, um parâmetro de difícil obtenção em comparação aos dados requeridos para aplicação do balanço hídrico. O balanço hídrico também tem a vantagem de fornecer estimativas especializadas, diferentemente de outros métodos como o WTF ou o método do deslocamento da curva de recessão (Recession Curve Displacement Method - RCDM). No método do WTF, as estimativas são referentes a uma área restrita ao entorno dos poços de monitoramento. No RCDM, obtém-se a recarga média em toda a bacia, não sendo possível identificar como a recarga ocorre espacialmente $\mathrm{e}$ individualmente em diferentes usos do solo.

\section{REFERÊNCIAS}

ALLEN, G.R.; PEREIRA, L.S.; RAES, D; SMITH, M. FAO Irrigation and Drainage Paper n. 56. Food and Agriculture Organization of the United Nations, 1998.

DINGMAN, S. L. Physical Hydrology. 3 edition ed. Waveland Press, Inc., 2014.

NATHAN, R.J., MCMAHON, T.A. Evaluation of Automated Techniques for Baseflow and Recession Analysis. Water Resources Research, v. 26, n. 7, p. 1465-1473, 1990.

GASTMANS, D.; KIANG, C.H; HUTCHEON, I. Stable isotopes $(2 \mathrm{H}, 18 \mathrm{O}$ and 13C) in groundwaters from the northwestern portion of the Guarani Aquifer System (Brazil). Hydrogeol. J. v. 18, p. 1497-1513, 2010.

GASTMANS, D; KIANG, C.H. Avaliação da hidrogeologia e hidroquímica do Sistema Aquífero Guarani (SAG) no estado do Mato Grosso do Sul. Águas Subt., v. 19, n. 35-48, 2005.

GOMES, L.H. Determinação da recarga profunda na bacia-piloto do Ribeirão da Onça em zona de afloramento do Sistema Aquífero Guarani a partir de Balanço hídrico em zona saturada. São Carlos-SP,
2008. 167p. (Dissertação de Mestrado). Universidade de São Paulo

HEALY, R; COOK, P. Using groundwater levels to estimate recharge. Hydrogeol. J., v. 10, p. 91-109, 2002.

HEALY, R.W.; WINTER, T.C.; LABAUGH, J.W; FRANKE, O. Water budgets: Foundations for effective water-resources and environmental management. U.S. Geological Survey Circular, 1308, 2007. 90p.

LUCAS, M.C.; GUANABARA, R.C; WENDLAND, E. Estimating groundwater recharge in the outcrop area of the Guarani Aquifer System. Bol. Geol. Min., v. 123, p. 311-323, 2012.

MACHADO, R. E.; MATTOS, A. Construção e instalação de um lisímetro com sistema de drenagem. Revista Brasileira de Agrometeorologia, Santa Maria, v. 9, n. 1, p. 147-151, 2001.

MACHADO, A. R.; WENDLAND, E.; KRAUSE, P. Hydrologic Simulation for Water Balance Improvement in an Outcrop Area of the Guarani Aquifer System. Environmental Processes, v. 3, n. 1, p. 19-38. doi: 10.1007/s40710-016-0128-4, 2016.

MEDEIROS, P.V.; MARCUZZO, F.F.N.; YOULTON, C; WENDLAND, E. Error autocorrelation and linear regression for temperature-based evapotranspiration estimates improvement. J. Am. Water Resour. Assoc., v. 48, p. 297-305, 2012.

MELO, D.C.D. Estimativa de impacto de mudanças climáticas nos níveis do aquífero livre em zona de recarga do Sistema Aquífero Guarani. São Carlos-SP, 2013. 180p. (Dissertação de Mestrado). Universidade de São Paulo.

OEA - ORGANIZAÇÃO DOS ESTADOS AMERICANOS. Programa estratégico de ação do projeto de proteção ambiental e desenvolvimento sustentável do Aquífero Guarani. Relatório Técnico, 2009.

SCANLON, B.R.; KEESE, K.E.; FLINT, A.L.; FLINT, L.E.; GAYE, C.B.; EDMUNDS, W.M.; SIMMERS, I; COOK, P. Global synthesis of groundwater recharge in semiarid 
and arid regions. Hydrol. Process, v. 20, p. 3335-3370, 2006.

SRACEK, O; HIRATA, R. Geochemical and stable isotopic evolution of the Guarani Aquifer System in the state of Sao Paulo, Brazil. Hydrogeol. J., v. 10, p. 643-655, 2002.

WENDLAND, E. Implementação de pacote computacional SPA (Simulação de Processos em Aquíferos). Universidade de São Paulo, 2003. (Relatório Técnico de Pesquisa FAPESP 02/09696-3)
WENDLAND, E; RABELO, J.L. Incertezas nos modelos de fluxo subterrâneo. R. Bras. Rec. Hídric., 15: 147-160, 2010. 